\title{
Automatic Control Technology Application on Friction Materials Industry \\ Meizhi Huang
}

Wuhan Huaxia Uninveristy of Technology, 430056

Key words: friction material, brake pad, process, automatic control, stability

\begin{abstract}
Friction materials need to be manufactured into brake pad and installed on vehicles. It is one component of vehicle, this industry need to meet automotive industry quality requirement like process stability and capability, so more and more automatic control technology is applied on friction material industry.
\end{abstract}

\section{Introduction}

In recent decades, China friction industry is blooming since vehicles volumes are increased rapidly. More and more friction materials companies produce brake pads for both OE and AM globally [1]. Brake pads are one important and safe part for vehicle, and its quality and process capability need to meet the automotive industry quality requirement. In friction material industry, more and more automatic control technology is used to improve productivity, process stability and process capability, furthermore, automatic control technology is used for testing in this industry as well.[2]

\section{Brake pad manufacturing process and its automatic control technology}

Friction materials is a kind of compounding material, which contains lots of chemicals ----resin and rubber acts bonding agency, metal oxides act as abrasive, graphite and metal sulfides act as lubricants. There is metal, fiber and filler as well [3]. Friction material manufacturing process is to ensure such compounding material can be produced into brake pads, which has good friction, good life time and good noise behavior. The whole process includes mix preparation, back plate preparation, pressing, curing, grinding, scorching, powder painting and final assembling [4]. In each process of friction materials manufacturing, more and more automatic control technology is used to avoid the deviation cause by manually operation. 


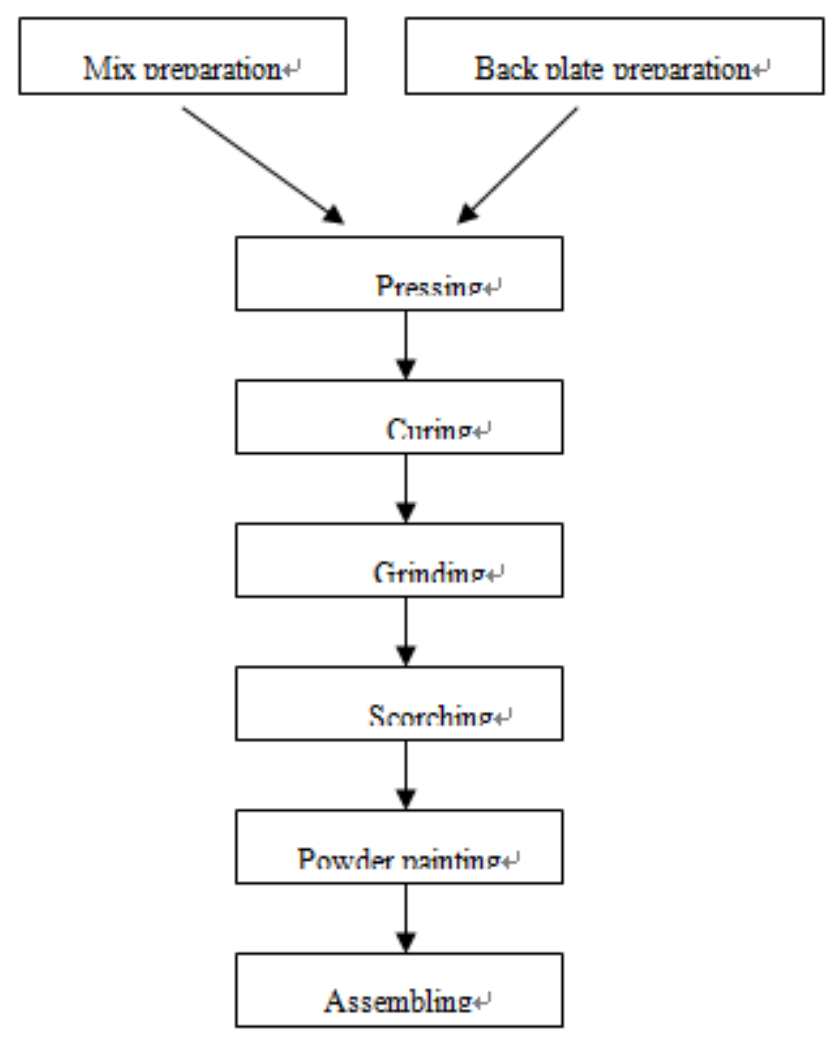

Figure 1. Friction materials manufacturing process

Mix preparation and its automatic control. Mix preparation is to weigh and load the chemicals according to friction materials formulation. It is import to keep the right content and sequence of each chemicals of formulation, otherwise, the performance for brake pad will be influenced significantly. Compare to former method, currently, bar code scanning is used to identify the right chemicals instead of by operator, this is the first step to follow the right action, then weighing is control by program of weighing system, the chemical and its weight is controlled and checked by the program, otherwise, next weighing cannot be done. This automatic control system can avoid the mistake from manually method. After weighing, mixing process is also controlled by program of mixer. In mixing process, mixing time, speed of plow and chopper are keys for this process. Automatic control program can ensure the process is running with right instruction.

\section{Back plate preparation and its automatic control}

Back plate preparation is cleaning the back plate, which is steel, and then doing sand blasting and glue rolling or spraying on back plate, finally, dry the glue. The glue coverage on back plate is very important for this process, since it will influence the bonding of friction material and back plate. How to check the glue coverage on back plate is the key of this process. Currently, automatic control technology is used in back plate preparation as well. It is scanning and video system. After glue rolling or spraying on back plate, it will pass through this system, and then it will check the coverage of glue on back plate. If it is not OK, the defects will be kicked out and it will not flow to next step. Compare to manually operation, the back plate will $100 \%$ check during this process and the standard is fixed. It can avoid miss check and deviation out of different operator. 
Pressing and its automatic control. Pressing is one important process of friction materials whole manufacturing process. Some key physical properties need to be achieved and control in this process. In traditional operation of this process, operator weighs the mix, load the mix into cavity of tooling, distribute the mix even in the cavity, put back plate, then pressing, and take out the pads and clean the tooling. All these actions are done by manually. There is this and that deviation with manual operation, and it will influence the physical properties and cause some quality problem. The process stability and capability is also lower with manual operation. Now, more and more automatic control technology is integrated with the pressing machine. With such automatic control machine, mix can be weighed automatically, mix loading and distribution is done automatically, then back plate feeding is done automatically, then pads unloading and tooling cleaning is done automatically. With such automatic control technology, not only productivity is improved, but also process stability and capability is improved. Furthermore, to improve the automation and productivity, there is kind of round table press is used in friction material industry. Such kind of round table press including several working station, one station for mix loading, one for mix distribution, one for pressing, one for pad unloading, these four working station can work at the same time, and move round to next step without any waiting time. [5]

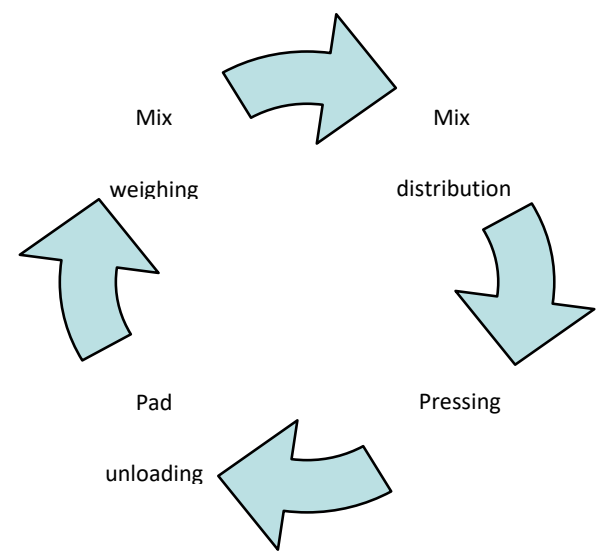

Figure 2 round table pressing schematic

After pressing, the brake pads should be cured. This process is let the chemicals react under temperature with enough time. It can improve the strength of friction material and meet the performance requirement of brake pads. In former time, after pressing thousands of pads, then put it together in racks and cure in heating oven. Such method need more and more ovens, and the temperature even within the oven sometimes cause quality problem. In recently, there is one kind of continuous oven with more accurate temperature control by program. After pad pressing, it can flow to the continuous oven, the pads move slowly in the continuous oven. So from overall process, after pressing, the pad is flowing without any stop. After curing, the chemicals of pads reaction are finished. Then brake pad need to grind to the shape according to drawing, which including surface grinding to target thickness, chamfering and slotting for the configuration. This process need several steps separately - grinding surface, then slotting, finally slotting. Currently, grinding process is more automatic and integrated. Surface grinding, chamfering and slotting can be done within one machine after setup the control program in the machine. It improves the productivity a lot and also ensure the configuration is more accurate since the automatic control. Scorching 
process is the process to do friction materials surface treatment at high temperature to reduce the initial effect; otherwise, the braking is not so efficient. During this process, the organic material is burned out at high temperature or high energy. Hot plate technology is used before, however, temperature control is challenge of this process. It causes some overheating and cracks problem for the pads, or some too slight scorching for brake pad and influence the braking performance. Now there is infrared technology is used in this process. Through the infrared energy control, it is easy to get the idea scorching. It also reduces the crack cause by overheating. They are cycles in which the static (very low speed) output of a braking system is measured. Static properties of brakes are usually measured to simulate how the brake will perform with the parking brake engaged, or simply the static friction level of the service brake. The automatic control is easy to adjust the infrared energy according to different pads requirement. Compare to traditional scorching, it is easy for production changeover and it is improve productivity a lot. Powder painting process is to apply anticorrosion powder on the back plate. This process was done by manually before. Then there is powder painting thickness problem, which cause the back plate rust. Now, automatic powder painting machine is used in this process as well. It make the thickness of powder painting is more even and also good appearance. After finish powder painting, brake pads need to be applied shim, clips, wear indicator and so on.

For such accessories application, automatic control technology is used as well to make the process controllable and stable.

\section{Conclusion}

Go through the whole process of friction materials manufacturing, more and more automatic control technology is used [6]. It makes the production more automatic. It reduces the deviation out of manual operation and improves the process stability and process capability. It also improves the quality of product out this friction material industry.

Along with the progress of automatic control technology, it will be upgrade friction material industry and widely used in the future.

\section{Reference}

[1] T.S. Wang, The character of European friction materials advanced technolog. 2002 friction material and sealing material exhibition technical paper.

[2] S.J. Kim, K.S.Kim, H. Jang, Optimization of manufacturing parameters for a brake lining using Taguchi method, J.Mater. Proc. Technol. 136 (2003) 202 208.

[3] J. Bijwe, Composites as friction materials: recent development in non-asbestos fiber reinforced friction materials - a review, polym. Comp. 18(3) (1997), 378 - 396.

[4] Y.Sasaki, M.Yanagi, process for producing friction material, patent, 2000.

[5]Pressing machine company introduction.

[6] Friction material manufacturing companies introduction 